September 1992

\title{
DISCRETE TO CONTINUOUS-TIME CROSSOVER DUE TO ANISOTROPY IN DIFFUSION-LIMITED TWO-PARTICLE ANNIHILATION REACTIONS
}

\author{
Vladimir Privman \\ Department of Physics, Clarkson University, Potsdam, New York 13699-5820, USA
}

PACS: 05.70.Ln, 68.10.Jy, 82.20.Mj

Key Words: Diffusion-Limited Reactions, Anisotropic Hopping, Exact Solutions, Continuum Limit

Running Title: Anisotropy in Diffusion-Limited Reactions

\begin{abstract}
Diffusion-limited reaction $\mathrm{A}+\mathrm{A} \rightarrow$ inert with anisotropic hopping on the $d=1$ lattice, is solved exactly for a simultaneous updating, discrete time-step dynamics. Diffusion-dominated processes slow down as the anisotropy increases. For large times or large anisotropy, one can invoke the appropriate continuum limits. In these limits the effects of the anisotropy on variation of particle density can be absorbed in time rescaling. However, in other regimes, when the discreteness of the time steps is nonnegligible, the anisotropy effects are nontrivial, although they are always quite small numerically.
\end{abstract}




\section{Introduction}

Recent studies of fluctuation effects in reaction-diffusion systems have been focused largely on exact or asymptotic continuum-limit analyses of low-dimensional, $d=1$ and $d=2$ models of the simplest reactions such as $\mathrm{A}+\mathrm{A} \rightarrow$ inert, $\mathrm{A}+\mathrm{A} \rightarrow \mathrm{A}$, etc. [112]. Particle input, fragmentation, and multiparticle reactions have been considered as well $[4,8,12-13]$. Fluctuation effects are significant in low dimensions; diffusion-limited reactions then show variation of the particle density at large times which is different from the predictions of the chemical rate-equation approach (mean-field theory). In this work we initiate studies of the effects of anisotropy in the hopping rates on lattice reactiondiffusion models. Specifically, we derive exact results for the reaction $\mathrm{A}+\mathrm{A} \rightarrow$ inert, in $d=1$.

Systems of hard-core particles show interesting novel density fluctuations associated with hopping rate anisotropy, such as shock-waves, etc. Recent studies [14-16] have yielded a variety of information pertinent to these phenomena, with most explicit results limited to $d=1$ models. We note however that the few simplest, solvable reaction schemes in $d=1$ involve instantaneous reactions such as two-particle annihilation. These reactions are termed diffusion-limited. The hard core property cannot be combined with annihilation on encounter unless the latter occurs with probability $<1$. The partial-reaction probability models can only be studied numerically [10,13]. Added hopping anisotropy may also have a profound effect on reactions in nonuniform systems with, e.g., point-like sources of particles, sinks, walls, etc. Investigation of these effects is, however, outside the scope of the present study.

Here we focus on the third interesting effect of the hopping rate anisotropy: slowing down of the diffusion. Indeed, let us consider a single particle, hopping on a line with the rate $H$ per unit time, and with the fixed step length $\ell$ to the right and to the left. Let the hopping rate to the right be $(1+a) H / 2$, while that to the left be $(1-a) H / 2$. 
Here we assume $-1 \leq a \leq 1$. In the long-time regime when the continuum description can be used, the particle diffusion constant $D(a)$ will be

$$
D(a)=\left(1-a^{2}\right) \ell^{2} H / 4=\left(1-a^{2}\right) D(0)
$$

Of course the diffusion is superimposed on the drift with velocity $a \ell H$. Note however that while the time scale of the drift is set by the original time variable $t$, the time scale of the diffusional spread is set by the dimensionless variable

$$
\tau(a)=4 D(a) t / \ell^{2}=\left(1-a^{2}\right) t H
$$

where the coefficient 4 was introduced for convenience. Thus, diffusion-dominated processes get slowed down as the anisotropy increases.

Our reaction-diffusion model for the $d=1$ reaction $\mathrm{A}+\mathrm{A} \rightarrow$ inert, will be defined in Section 2, within the cellular-automaton, simultaneous updating formulation [11], with discrete time steps $\Delta t=1$. However, we will demonstrate (Section 4) that the model approaches the continuum-time reaction-diffusion kinetics when the "natural" time steps, $\Delta \tau=\left(1-a^{2}\right) H \Delta t$, vanish in the extreme anisotropy limit $|a| \rightarrow 1$. Exact solution will be obtained for all $a$ values, in Section 3. The $a=0$ case corresponds to the isotropic reaction solved in the cellular-automaton variant in [11]. The $|a|=1$ limiting

solution turns out (Section 4) to be identical with the result obtained in the isotropic case for the continuum-time Glauber-dynamics variant of the reaction $\mathrm{A}+\mathrm{A} \rightarrow$ inert, solved in $[5,9]$. Section 4 also contains a summarizing discussion.

\section{Definition of the Model}

We consider particles hopping on a linear $x$-lattice of spacing $\ell$. The dynamics is 
defined to have the evolution in time steps $\Delta t=1$. At a given time $t=0,1,2, \ldots$, we assume that the reacting particles occupy, with certain average density $\rho(t)$, odd lattice sites, $x / \ell= \pm 1, \pm 3, \ldots$, at even times $(t=0,2,4, \ldots)$, and even lattice sites $(x / \ell=0, \pm 2, \ldots)$ at odd times $t=1,3, \ldots$. There can be 0 or 1 particles at each site (of the relevant sublattice). At each time step $t \rightarrow t+1$, each of the particles randomly hops one lattice spacing to the right $(+\ell \hat{x})$ or to the left $(-\ell \hat{x})$, with respective probabilities $(1+a) / 2$ and $(1-a) / 2$. The hopping events are independent. However, at those sites which at time $t+1$ received 2 particles, the pairs "annihilate," i.e. the occupation number is immediately reduced to 0 . It is obvious that this rule decouples the even and odd sublattices at alternative time steps, - the reason for our restriction to the even/odd sublattices as described earlier.

Quantities such as the particle density per site $\rho(t)$ are calculated by averaging not only over the hopping direction choices but also over the initial particle distribution at time $t=0$. In order to have an exactly solvable model, here as for the isotropic case $a=0[9,11]$, one has to consider a particular choice of the initial particle distribution: randomly occupied (odd) sites with the average initial density $\rho(0)=1 / 2$ (per oddsublattice site).

The root to exact solutions of annihilation models $\mathrm{A}+\mathrm{A} \rightarrow$ inert, involves the transformation $[5,9,11]$ from the hopping particle dynamics to the dual-lattice stochastic spin dynamics. In order to introduce the dual lattice systematically, let us describe the original particle system in the following somewhat unusual way: the occupancy variables $m_{j}(t)$ will take values -1 or 1 , for occupied sites $x=j \ell$ or empty sites, respectively. Thus the particle density is

$$
\begin{aligned}
\rho(t)=\langle & {\left.\left[1-m_{j}(t)\right] / 2\right\rangle . } \\
& -4-
\end{aligned}
$$


Note that the density does not depend on $x=j \ell$ because the initial conditions and stochastic hopping rules, - average over both being denoted by $\langle\ldots\rangle$, - are translationally invariant.

We now introduce the dual-lattice spins $\sigma_{j}(t)= \pm 1$, on the even sublattice for even times, and on the odd sublattice for odd times. Thus, these spins are located in the interstices $x=j \ell$ between the allowed particle-location sites at $x=(j \pm 1) \ell$. Formally, the spin values are defined by the infinite products

$$
\sigma_{j}(t)=\prod_{i=0}^{\infty} m_{j+2 i+1}(t)
$$

although with some care in definitions, products up to some fixed $m_{J}(t)$ could be used as well. This definition implies

$$
m_{j}(t)=\sigma_{j-1}(t) \sigma_{j+1}(t)
$$

so that the density is given by

$$
2 \rho(t)=1-\left\langle\sigma_{j-1}(t) \sigma_{j+1}(t)\right\rangle=1-G_{2}(t)=G_{0}(t)-G_{2}(t),
$$

where we define the two-point spin-spin correlation function

$$
G_{n}(t)=\left\langle\sigma_{i}(t) \sigma_{i+n}(t)\right\rangle
$$

which only depends on the separation $|n|$ due to translational invariance. We will consider this function for $n=0,2,4, \ldots$.

While the stochastic dynamics of the original occupancy variables $m_{i}(t)$ is nonlinear, it reduces to a linear dynamical rule for the dual spins. This can be shown directly 
by first introducing the particle dynamics. However, the steps involved are cumbersome. A simpler approach is to define the linear spin dynamics first and check that the particle hopping and annihilation are correctly represented by it. Thus, let us introduce random variables $\xi_{i}(t)$ taking on values 1 or 0 , with respective probabilities $(1-a) / 2$ and $(1+a) / 2$, where $a$ is the hopping bias. Then the dynamical rule is

$$
\sigma_{i}(t+1)=\left[1-\xi_{i}(t)\right] \sigma_{i-1}(t)+\xi_{i}(t) \sigma_{i+1}(t)
$$

Having $i$ as a spin site at time $t+1$ suggests that at time $t$ the site $i$ was a possible hopping-particle location site; recall our sublattice rules. We now identify the random variable values $\xi_{i}(t)=0$ or 1 as the decisions to move the particle to the right or to the left, respectively, provided of course that there is a particle at the site $x=i \ell$ at time $t$. Let us also define more natural particle occupancy variables,

$$
n_{i}(t)=\left[1-m_{i}(t)\right] / 2
$$

which take on values 0 (empty site) or 1 (occupied site), and average to $\rho(t)$.

When we calculate $m_{i}(t+1)$ in terms of the $m_{j}$ variables at the preceding time, $t$, by substituting (2.6) in the $t+1$ version of (2.3), the relation is no longer linear. The source of the nonlinearity is the term proportional to $\sigma_{i-2} \sigma_{i+2} \equiv m_{i-1} m_{i+1}$. After some algebra, we get, in terms of the $n_{i}$ variables,

$n_{i}(t+1)=\xi_{i+1}(t) n_{i+1}(t)+\left[1-\xi_{i-1}(t)\right] n_{i-1}(t)-2 \xi_{i+1}(t) n_{i+1}(t)\left[1-\xi_{i-1}(t)\right] n_{i-1}(t)$.

It is obvious that this nonlinear dynamics corresponds to the particle-hopping rules as specified originally. Indeed, the first term adds 1 to $n_{i}(t+1)$ if there was a particle 
at site $i+1$ at $t$, and if it hopped to the left. The second term adds 1 if there was a particle at $i-1$ and it hopped to the right. Finally, the last, "reaction" term, which is $(-2)$ times the product of the first two, "diffusion" terms, sets the resulting value to zero if both diffusion terms were 1, i.e., if two particles hopped to $i$ in the time step $t \rightarrow t+1$. Unfortunately, we found no other nontrivial quadratic dynamical rules that can be linearized by the dual-spin transformation or its simple generalizations. Thus, the reaction $\mathrm{A}+\mathrm{A} \rightarrow$ inert is the only one solvable by the dual-spin method. For instance, if we replace $(-2)$ by $(-1)$ in $(2.8)$ to have the reaction scheme $\mathrm{A}+\mathrm{A} \rightarrow \mathrm{A}$, then the dualspin method fails. The latter reaction can be solved by another method, however; see $[12]$.

Finally, we note that the hopping rate of a single particle is $H=1$. Thus, the dimensionless time variable $\tau$ is given by

$$
\tau=\left(1-a^{2}\right) t
$$

\section{Exact Result for the Particle Density}

The initial particle distribution was selected to correspond to the random \pm 1 assignment of the initial $(t=0)$ spin values. Averaging (2.6) over the stochastic variables $\xi$ then leads to the conclusion that $\left\langle\sigma_{i}(t)\right\rangle=0$ for all times $t \geq 0$. The two-point correlation function, however, is nontrivial. Forming the product suggested by the definition (2.5), we obtain, after averaging and some algebra,

$$
G_{n}(t+1)-G_{n}(t)=\frac{1-a^{2}}{4}\left[G_{n-2}(t)-2 G_{n}(t)+G_{n+2}(t)\right] .
$$

This relation applies only for $n=2,4,6, \ldots$, and it must be solved subject to the 
boundary condition

$$
G_{0}(t \geq 0)=1
$$

as well as the initial condition

$$
G_{n>0}(0)=0
$$

Let us introduce the generating functions

$$
B_{n}(v)=\sum_{t=0}^{\infty} v^{t} G_{n}(t),
$$

as well as the generating function for the density,

$$
R(v)=\sum_{t=0}^{\infty} v^{t} \rho(t)=\frac{1}{2}\left[B_{0}(v)-B_{2}(v)\right]
$$

where the last equality follows from (2.4). Note that we have

$$
B_{0}(v)=\frac{1}{1-v}
$$

while the $n>0$ generating functions satisfy the recursion

$$
\frac{B_{n}}{v}-B_{n}=\frac{1-a^{2}}{4}\left(B_{n-2}-2 B_{n}+B_{n+2}\right) .
$$

Each term in (3.7) follows directly from summation of the appropriate term in (3.1).

This second-order difference equation is quite standard. Only one out of two independent solutions behaves regularly for large $n$, in the vicinity of $v=0$. The solution takes the form 


$$
B_{n}(v)=B_{0}(v)[b(v)]^{n / 2},
$$

where the appropriate function $b(v)$ is found directly from (3.7),

$$
b(v)=1+\frac{2(1-v)}{\left(1-a^{2}\right) v}\left[1-\sqrt{1+\frac{\left(1-a^{2}\right) v}{1-v}}\right] .
$$

The generating function for the density then follows as

$$
R(v)=\frac{1}{\left(1-a^{2}\right) v}\left(\frac{\sqrt{1-a^{2} v}}{\sqrt{1-v}}-1\right)
$$

where we made several algebraic steps to prepare the resulting expression for the Taylor series expansion to yield the density:

$$
\left(1-a^{2}\right) \rho(t)=\frac{(2 t+2) !}{2^{2 t+2}[(t+1) !]^{2}}-\sum_{n=0}^{t} \frac{(2 n) !(2 t-2 n) ! a^{2 n+2}}{2^{2 t+1} n !(n+1) ![(t-n) !]^{2}} .
$$

\section{Special Limits and Discussion}

In this section we consider three limiting cases in which the solution (3.11) assumes less cumbersome forms. First, we note that for the symmetric- hopping case, $a=0$, only the first term in (3.11) survives. Since $\tau=t$ for $a=0$, we have

$$
\rho_{a=0}(\tau)=\frac{(2 \tau+2) !}{2^{2 \tau+2}[(\tau+1) !]^{2}} .
$$

This form provides a convenient analytic continuation to all $\tau \geq 0$, shown as the lower

solid curve in Figure 1. However, the reader should note that the actual time-dependence for $|a| \neq 1$ is always in discrete time steps. 
Next, consider the limit $|a| \rightarrow 1$, i.e., extreme anisotropy and slow diffusion. The "natural" time steps $\Delta \tau=\left(1-a^{2}\right)$ then become small and the time-dependence approaches a continuous function of $\tau$. As a result, the discrete-time difference on the left-hand side of (3.1) can be replaced by the derivative $\left(1-a^{2}\right) \partial G_{n}(\tau) / \partial \tau$. The resulting equations are then identical, up to minor notational changes, to those obtained in the continuum-time Glauber-dynamics formulation [5,9] of the (isotropic) reaction $\mathrm{A}+\mathrm{A} \rightarrow$ inert. They can be solved, for instance, by the Laplace Transform method. We only present the final expression for the density,

$$
\rho_{|a|=1}(\tau)=\frac{1}{2} e^{-\tau / 2}\left[I_{0}(\tau / 2)+I_{1}(\tau / 2)\right],
$$

where $I_{n}$ are the standard Bessel functions. The upper solid curve in Figure 1 corresponds to (4.2).

Finally, we note that for large times both the temporal and spatial variation of the two-point correlation function can be regarded as slow and differences replaced by derivatives. Specifically, the second-difference expression on the right-hand side of (3.1) is replaced by the second spatial-coordinate derivative. The detailed derivation of this continuum limit and solution for two-point correlations are presented, e.g., in [11]. In this limit, the hopping anisotropy is fully absorbed in the rescaling of time from $t$ to $\tau$. We only quote the result for the density

$$
\rho(\tau \gg 1) \simeq \frac{1}{\sqrt{\pi \tau}} .
$$

This function is shown by the dashed line in Figure 1. It is asymptotic to the solid curves for large $\tau$, as well as to the full result (3.11).

The full expression (3.11) is more complicated than the limiting cases just discussed. 
The density, defined for discrete $\tau$-values, — integral multiples of $\left(1-a^{2}\right)$, — lies between the two limiting solid curves shown in Figures 1 and 2. For fixed $t$, the $\tau$ values decrease as $|a| \rightarrow 1$. Thus, the crossover from the $a=0$ discrete values to the $|a|=1$ continuous $\tau$-dependence occurs for fixed $t$ as shown in Figure 2. The points in Figure 2 correspond to $t=2$ and

$$
a=0.00,0.05,0.10, \ldots, 0.95
$$

note that the symbols for the points corresponding to $a=0$ and $1 / 20$ largely overlap in the figure.

In summary, we derived exact results for a two-particle annihilation reaction with anisotropic hopping on a $d=1$ line. The main effect of the anisotropy is to slow down the diffusion-dominated processes, and it can be largely absorbed in the redefinition of the time scale. However, in the regimes where the discreteness of time steps is nonnegligible, i.e., for short times and nearly isotropic hopping, the functional form of the density depends on the hopping anisotropy parameter $a$ explicitly. The discretetime-step reaction proceeds slightly faster than the continuous-time reaction, though the difference is numerically quite small.

The author wishes to thank M. Barma, M.L. Glasser and S. Nicolis for helpful comments and suggestions. 


\section{REFERENCES}

1. M. Bramson and D. Griffeath, Ann. Prob. 8, 183 (1980).

2. D.C. Torney and H.M. McConnell, J. Phys. Chem. 87, 1941 (1983).

3. K. Kang, P. Meakin, J.H. Oh and S. Redner, J. Phys. A17, L665 (1984).

4. T. Liggett, Interacting Particle Systems (Springer-Verlag, New York, 1985).

5. Z. Racz, Phys. Rev. Lett. 55, 1707 (1985).

6. A.A. Lushnikov, Phys. Lett. A120, 135 (1987).

7. M. Bramson and J.L. Lebowitz, Phys. Rev. Lett. 61, 2397 (1988).

8. Review: V. Kuzovkov and E. Kotomin, Rep. Prog. Phys. 51, 1479 (1988).

9. J.G. Amar and F. Family, Phys. Rev. A41, 3258 (1990).

10. L. Braunstein, H.O. Martin, M.D. Grynberg and H.E. Roman, J. Phys. A25, L255 (1992).

11. V. Privman, J. Stat. Phys. (1992), in print.

12. D. ben-Avraham, M.A. Burschka and C.R. Doering, J. Stat. Phys. 60, 695 (1990), and references therein.

13. V. Privman and M.D. Grynberg, J. Phys. A (1992), in print, and references therein.

14. Z. Cheng, J.L. Lebowitz and E.R. Speer, Comm. Pure Appl. Math. XLIV, 971 (1991).

15. S.A. Janowsky and J.L. Lebowitz, Phys. Rev. A45, 618 (1992).

16. F.J. Alexander, Z. Cheng, S.A. Janowsky and J.L. Lebowitz, J. Stat. Phys. 68, 761 (1992). 


\section{FIGURE CAPTIONS}

Figure 1: Particle density as a function of the dimensionless time variable $\tau$. Lower solid curve: $a=0$, the actual densities correspond to integer $\tau$ values; see Section 4 . Upper solid curve: $a= \pm 1$. Dashed curve: the $\tau \gg 1$ asymptotic form.

Figure 2: The points illustrate the crossover from $a=0$ to $|a|=1$, for a fixed time,

$t=2$. The $a$ values are given in Section 4 ; see (4.4). The solid curves are the same as in Figure 1. 